\title{
Field Mapping in Semiconductors by Off-axis Electron Holography: From Devices to Graphene and Single Dopant Atoms
}

\author{
David Cooper $^{1}$, Jean-Luc Rouvière ${ }^{2}$ and Rafal Dunin-Borkowski ${ }^{3}$ \\ 1. CEA-LETI, Minatec, 17 rue des Martyrs, 38054 Grenoble Cedex 9, France \\ 2. CEA-INAC, Minatec, 17 rue des Martyrs 38054 Grenoble Cedex 9, France \\ 3. Ernst-Ruska Center for Microscopy and Spectroscopy with Electrons, Research Centre Julich,D-52425 \\ Julich, Germany
}

Off-axis electron holography is an electron microscopy-based technique that allows the electrostatic and magnetic fields in and around a specimen to be measured with nm-scale resolution. The continuous reduction in the size of semiconductor devices means that information about the distribution of strain fields and active dopants at a nanometre scale is required in order to understand how these properties affect their electrical performance. Indeed, the reduction in the size of these devices is in some ways beneficial for electron holography as the higher dopant concentrations that are used are less affected by artefacts from specimen charging and preparation. As these devices are miniaturised further, what was once known as a very high dopant concentration will be only a few atoms and the positions of these individual atoms will affect the properties of the device. As a consequence we are working towards the detection of single dopant atoms by off-axis electron holography.

In this presentation we will show how electron holography can be used to measure the strain and electrostatic potentials in a range of semiconductor devices across different scales of dimensions from large conventional silicon transistors to graphene sheets. By using a FEI Titan operated at $200 \mathrm{kV}$ and combined with back-side focused ion beam milling at low energies, it is now possible to perform routine systematic studies on semiconductor devices during development. Figure 1 shows a series of potential maps for individual electrically tested pMOS devices that have been processed using different gate architectures and dopant implantations. The potential maps have been compared to simulations and electrical tests to provide a comprehensive understanding of the how the dopants affect the device properties. Figure 2 shows the deformation measured in during this study for different devices measured by dark field electron holography confirming that the strain introduced by the nitride film is consistent in both of them. In this presentation we will briefly show how other strain mapping techniques such as nanobeam diffraction using precession have been used to confirm the results.

Finally we will show recent work that focuses on the ultimate performance of electron holography with the final goal of single dopant atom detection. Figure 3(a) shows a phase image of a boron nitride flake acquired using off-axis electron holography applied in high resolution mode using a FEI Titan Ultimate with an image corrector and a single biprism operated at $80 \mathrm{kV}$. The microscope has been operated in free lens control in order to have a fringe spacing of 0.35 Angstroms with a narrow hologram width in order to preserve the contrast of the fringes. Figure 3(b) shows a detail of (a) and (c) a phase profile extracted from the indicated region in (b) showing that the $\mathrm{B}-\mathrm{N}$ bond has been resolved.

[1] D Cooper et al, Semiconductor Science and Technology 28 (2013), p. 125013.

[2] D Cooper thanks the European Research Council for funding this work with the Starting Grant 325525 "Holoview". 

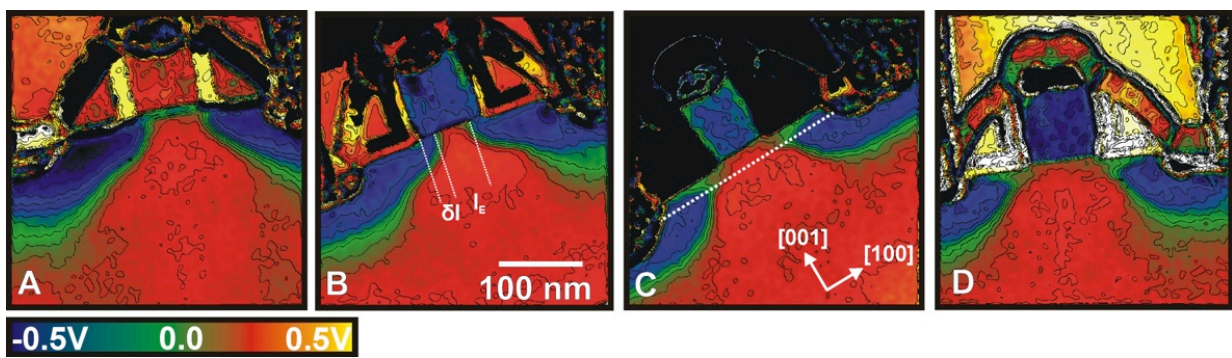

Figure 1. Maps of the changes in electrostatic potential arising from active dopants in differently processed nMOS devices.
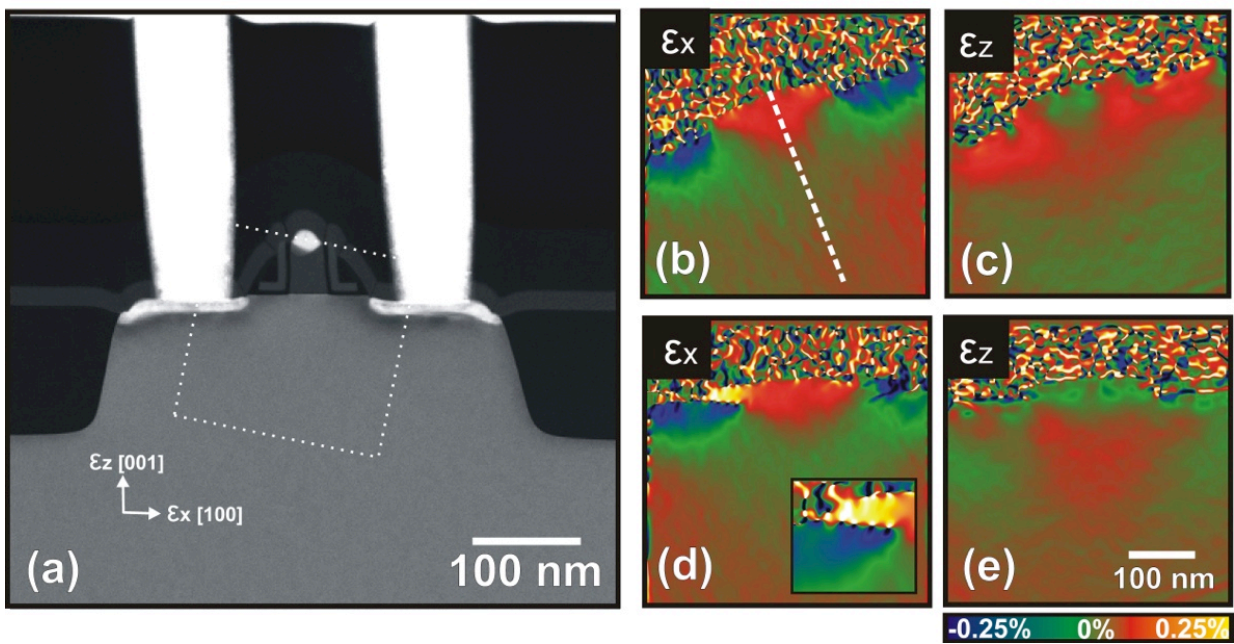

Figure 2. (a) STEM image of device. (b) In plane deformation map and (c) growth direction deformation map for an nMOS device and (d) the in plane and (e) growth deformation for a pMOS device.
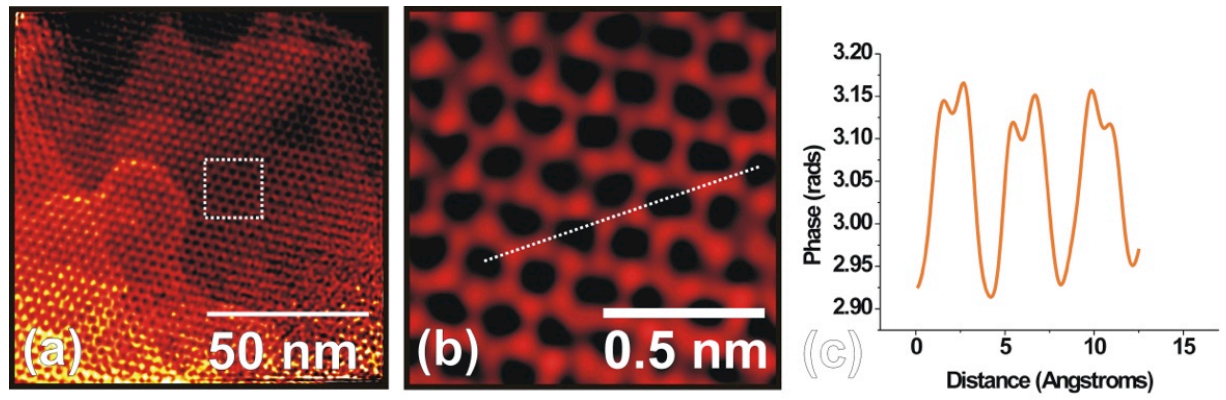

Figure 3. (a) Reconstructed phase image of a flake of boron nitride (b) detail from region indicated in (a). (c) Phase profile acquired across region indicated in (c) showing that the 1.4Angstrom-spaced B-N bonds have been resolved. 